\title{
Localization of a peripheral target during parametric adjustment of saccadic eye movements ${ }^{1.2}$
}

\author{
SAMUEL C. McLAUghLIN, Jr., MARTIN J. KELLY, Jr., ROBERT E. ANDERSON, ${ }^{3}$ AND TERESA G. WENZ ${ }^{4}$ \\ TUFTS UNIVERSITY
}

\begin{abstract}
The aim of the experiment was to find out whether saccadic eve movements have any effect on perceived visual directions. The method was to alter the parameters of the oculomotor system so that the eye movement made in response to a peripheral target was inappropriate to the retinal locus of its image. It was found that this procedure had no effect on the perceived location of the peripheral target; and it was concluded that a specific retinal locus is more or less rigidly associated with a corresponding visual direction, but not with a particular magnitude of ocular rotation.
\end{abstract}

If one is asked to estimate the length of a line, one's first reaction is to scan the line, fixating each end in turn, as if to measure it in terms of the amount of ocular rotation required to traverse it. A similar response can be seen when a carpenter or surveyor estimates a dimension. Watching his eye movements, one has the impression that the artisan is doing more than merely getting a clear image for each part of the scene to be analyzed: it looks very much as if the eye movements themselves are contributing something to the estimate of size or extent. One need not postulate, as Lotze did, that visual space is built up entirely from eye movements; but it seems not unreasonable to suppose that eye movements may contribute something to visual metric judgments and hence to perceived visual directions.

The recent discovery of a technique for eliciting parametric adjustment of saccaddic eye movements (McLaughlin, 1967) provided a way to test that possibility. The technique is to have $S$ make a series of eye movements from one target to another-say, from Target A to Target B. Prior to each eye movement, while $S$ is fixating Target A, B is at some fixed position with respect to A-say, 10 deg to the left. During each eye movement, Target B is displaced some arbitrary distance toward A. As a result, the eye tends to overshoot the final position of $B$. This sequence of events is repeated on each eye movement of the series: B starts out at $10 \mathrm{deg}$ left and undergoes exactly the same displacement each time. The result is that the overshoot gradually diminishes, until (usually after 8 or 10 eye movements) the eye goes directly to the final (displaced) position of Target $B$. This happens despite the fact that Target $B$ is unequivocally visible to $S$ while he is fixating Target A.

In this procedure, Target $B$ is imaged on the same retinal locus ( $3 \mathrm{~cm}$ to right of the fovea centralis) at the start of each eye movement, whereas the eye movement made in response to $B$ gradually changes. Hence, if eye movements have any effect on perceived visual directions, the procedure should bring about a change in the perceived location of Target $B$ as seen in peripheral vision. The purpose of the present experiment was to find out if such a change occurs.

\section{Subjects}

\section{METHOD}

Subjects were three adults: one female and two males. All three had extensive experience in this and related experiments over a period of 6-12 months.

\section{Apparatus}

The S's head was fixed in position by a bite plate bearing his dental impression, rigidly attached to the experimental table. The left eye was covered with an eye patch.

Eye position was monitored using the principle described by Torok et al in 1951 (Fig. 1). In this method, matte reflectance from an area on the surface of the eyeball is imaged on a photoelectric device. If the area selected is near the horizontal meridian of the eye, and if it contains portions of both iris and sclera, then the output of the photodetector will vary with rotational position of the eye about a vertical axis.

In our application of this principle (Fig. 2), a spherical reflector $30 \mathrm{~cm}$ in diameter was placed with its back surface $50 \mathrm{~cm}$ from the eye and with its optical axis in a horizontal plane $37 \mathrm{deg}$ to the right of the primary visual axis of S's right eye with that eye in its primary position. The eye was diffusely illuminated with deep red light (incandescant lamp, Wratten 89B filter). The reflector formed an image of the eye on a small screen $3 \mathrm{~cm}$ square. In the center of the screen was a slit $1 \mathrm{~mm}$ high and $8 \mathrm{~mm}$ wide. Directly behind the slit was a semiconductor photodiode.

The output of the photodiode circuit, after preamplification, was led to one amplifier of a dual-trace oscilloscope. During the experiment, the CRO tube was photographed at appropriate times with a Grass oscilloscope camera. The overall frequency response of this system was in excess of $100 \mathrm{~Hz}$. An eye movement of less than $1 \mathrm{deg}$ could be made to produce a $10 \mathrm{~cm}$ deflection of the oscilloscope trace, which suggests that the system may have been able to record an ocular rotation of less than $1 \mathrm{~min}$ arc. Figure 3 shows a typical linearity calibration.

The visual display (Fig. 4) consisted of two luminous targets (brightness approximately $20 \mathrm{~mL}$ ) seen against a dark background at a viewing distance of $33 \mathrm{~cm}$. Target A, 5 deg toward S's right, was $1.5 \mathrm{~mm}$ in diameter. B was $10 \mathrm{deg}$ to the left of A. Each target had cross-hairs for precise foveal fixation.

The displacement of Target $\mathrm{B}$ had to be completed during the fast portion of an eye movement which lasted only some $.04 \mathrm{sec}$. To achieve this degree of speed and precision, $B$ was never actually moved. Instead, a third target, $B^{\prime}$, identical to $B$, was mounted so that it appeared to $S$ to be $5 \mathrm{deg}$ to the right of $B$. Targets $A$ and $B$ were seen through a beam-splitting prism, and $B^{\prime}$ was seen via the prism. $B$ and $B^{\prime}$ were fluorescent lamps operated in such a way that they could be turned on and off in a few microseconds. Each was invisible to $S$ when it was turned off. At no time during the experiment were $B$ and $B^{\prime}$ on together.

Apparent displacement of $\mathrm{B}$ was accomplished by connecting the eye position signal to a solid-state Schmitt trigger. With the apparatus in "switching" mode, operation of the trigger by an eye

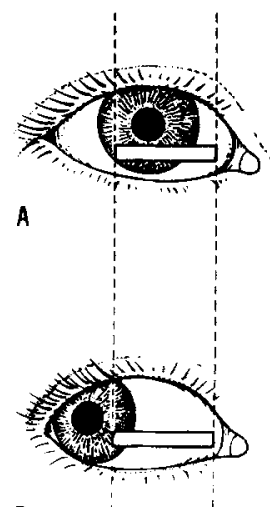

B

Fig. 1. Illustrating the principle of eye-movement monitoring by differential matte reflectance from iris (dark) and sclera (white). The area outlined by the rectangle is imaged on a photoelectric device. The output of the photodetector then varies with rotational position of the eye about a vertical axis. From Torok et al (1951). 


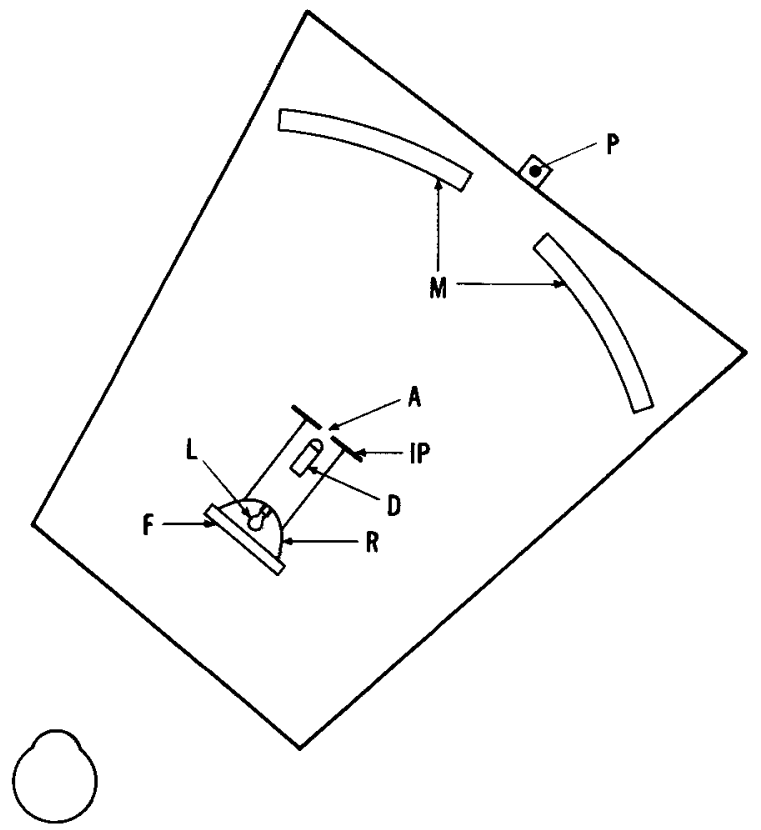

Right Eye

Fig. 2. Schematic diagram of eye movement detector in plan view. The spherical reflector $M$ forms an image of the eye on the image plate IP, in the center of which is a rectangular aperture $A$. Directly behind the aperture is a photodetector D. The light which illuminates the eye is emitted from the lamp $L$, focussed by the reflector $R$, and transmitted through the red filter $F$. Mirror, detector, and illumination system are mounted on a common base which pivots on $P$.

movement caused $B$ to turn off and $B^{\prime}$ to turn on during the eye movement. The switching delay was the pull-in time of a reed relay, approximately $1 \mathrm{msec}$.

The use of a peripheral target of large vertical extent was suggested by Dodge and Cline (1901) as a means of ensuring that the peripheral target does not fade from view prior to the change of fixation. The dark areas above and below the cross-hairs enabled $S$ to judge the vertical location of the cross-hairs even though the cross-hairs themselves could not be seen in peripheral vision. ${ }^{5}$

Directly underneath the top of the table was a pointer which $S$ could move in an are under Targets $A$ and $B$, using his right hand. The extreme right-hand limit of pointer travel was directly beneath Target A. The extreme left hand limit of pointer travel was more than 10 deg to the left of B. During the experiment, $S$ was not able to see his right hand or arm or any part of the pointer.

The pivot of the pointer was the vertical shaft of a potentiometer which was mounted under the bite plate, approximately on a plumb line with the center of S's right eye. A voltage was applied to the potentiometer, and the output of the potentiometer was connected to the second amplifier of the oscilloscope.

\section{Procedure}

Prior to the present experiment, each $S$ had extensive practice and instruction in (a) remaining motionless on the bite plate for at least $5 \mathrm{~min}$, (b) fixating precisely on the cross-hairs of whichever target he was looking at, (c) making a rapid and accurate change of fixation in response to the onset of the buzzer, and (d) maintaining fixation on $A$ while setting the pointer under the apparent position of $B$ or $B^{\prime}$.

A single experimental run consisted of four parts. Part 1 consisted of four eye movements from $A$ to $B$, each followed by a pointer setting under the apparent position of B. Part 2 consisted of 11 consecutive eye movements with $\mathrm{B}^{-\mathrm{B}^{\prime}}$ switching (no pointer settings). Part 3 consisted of four eye movements with B-B' switching, each followed by a pointer setting under the apparent position of B. Part 4 consisted of four eye movements from $A$ to $B^{\prime}$, each followed by a pointer setting under the apparent position of $\mathbf{B}^{\prime}$.

Prior to each eye movement in Part 1, S fixated Target A while B was visible to him in peripheral vision. On signal (a buzzer), he shifted his gaze toward B. Two sec after the buzzer had sounded, $B$ was turned off. There was a delay of several seconds during which $S$ was allowed to blink or to close his eyes. He then fixated $A, B$ came on, and he continued to fixate $A$ while setting the pointer under the apparent position of $B$. Each pointer setting started with the pointer under $A$; after each pointer setting, $S$ returned the pointer to its extreme right-hand position (under $A$ ).

In Part 2 , each eye movement started with $S$ looking at $A$ while B was visible to him in peripheral vision. When the buzzer sounded, he shifted his gaze toward B. During the fast portion of the eye movement, $B$ turned off and $B^{\prime}$ tumed on. Two sec after the buzzer had sounded, B' turned off. After the "blink" period, S again fixated $A$ and $B$ came on.

Part 3 consisted of four eye movements with switching, each followed by a pointer setting under the apparent position of $B$. As in Part 1, $S$ fixated A during each pointer setting.

Part 4 consisted of four ordinary eye movements from $A$ to $B^{\prime}$,
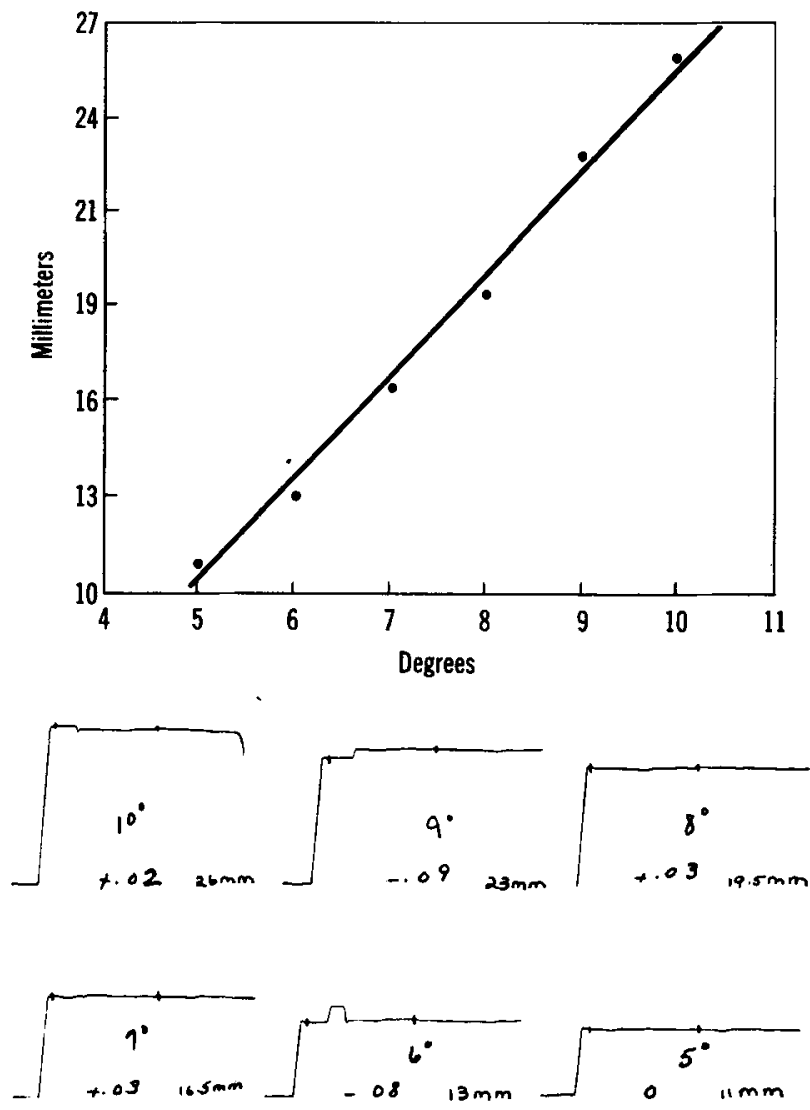

Fig. 3. Upper, a typical linearity calibration. Lower, the eye movement reconds on which the calibration is based. Each eye movement record shows a change of fixation from Target $A$ to a target at $10 \mathrm{deg}, 9 \mathrm{deg}$, etc. Each recond starts at the left and has a duration of 2.1 sec. A movement upward in the record represents an ocular rotation toward S's left. The $\mathrm{mm}$ measurements indicate the magnitude of the eye movement in $\mathrm{mm}$ on the original record, and are the ordinal values in the diagram. The algebraic (signed) numbers indicate the amount of overshoot in each initial saccade, and are not relevant to the calibration. 


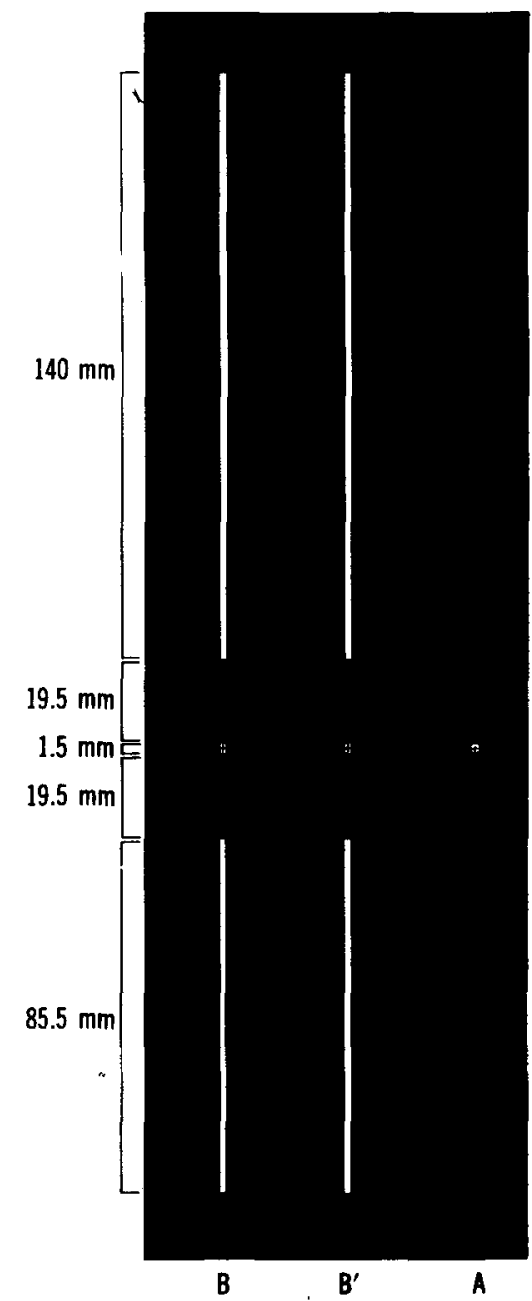

Fig. 4. Visual display. At the viewing distance used $(33 \mathrm{~cm}), 1 \mathrm{~mm}$ corresponds to $10 \mathrm{~min}$ arc and $6 \mathrm{~mm}$ corresponds to 1 deg. Targets $A$ and $B$ were seen through a beam splitting prism and $B^{\prime}$ was seen via the prism. $B$ and $B^{\prime}$ were never on together, and neither was visible to $S$ when it was turned off. During an eye movement from $A$ to $B, B$ was switched off and $B^{\prime}$ was switched on, thus inducing an overshoot with respect to $B^{\prime}$.

each followed by a pointer setting under the apparent position of $B^{\prime}$. Target $B$ was not on at any time during Part 4.

Each run was preceded and followed by six eye movements for calibration (Fig. 3). The $S$ remained on the bite plate without interruption throughout preliminary calibration, experimental run, and final calibration. There was no pause after any of the first three parts of a run. After final calibration, $S$ had at least $5 \mathrm{~min}$ off the bite plate before starting the next run.

The procedure and instructions for a single run were programmed on a 2-channel tape recorder. One channel carried verbal instructions which $S$ heard over a loud-speaker. The other channel carried 5 sub-channels of frequency-coded switching signals which controlled the entire apparatus. A single run (not including calibration) lasted $4 \mathrm{~min} 44 \mathrm{sec}$.

Each $S$ went through three runs in a single experimental session. In another experimental session, each $S$ went through three runs which were identical except that the Schmitt trigger was turned off, so that no switching occurred during any eye movement.

Analysis of eye movement records

\section{RESULTS}

A 10 deg change of fixation, with or without switching, usually consists of an initial saccade followed by one or more corrective eye movements. The datum of interest here is the magnitude of the initial saccade. To obtain this datum, the eye position $0.1 \mathrm{sec}$ after the mid-point of the initial saccade was taken as the terminal point of that saccade. The eye position $0.7 \mathrm{sec}$ later, or $0.8 \mathrm{sec}$ after the mid-point of the initial saccade, was assumed to represent foveal fixation of B or B'. The arithmetic difference between these two values was taken as the measure of the amount of overshoot or undershoot in the initial saccade. The magnitude of the initial saccade was calculated from this value, and was thus determined with reference to $B$ or $B^{\prime}$, not with reference to $A$.

\section{Statistical test of parametric adjustment}

This test was made by comparing two groups of eye movements: (a) all eye movements in Part 1 and additional A-B eye movements from runs with no switching, and (b) all eye movements after the first in Part 2 with switching, and all in Part 3 with switching. The first group was assumed to represent normal change of fixation, and the second was assumed to represent the effect, if any, of B-B' switching. Combining data for all three Ss. there were 126 eye movements in each of the two groups.

In the first group (no parametric adjustment), the mean initial saccade was $9.43 \mathrm{deg}(\mathrm{SD}=0.90 \mathrm{deg})$. In the second group (parametric adjustment) the initial saccade was $5.80 \mathrm{deg}$ $(\mathrm{SD}=1.23 \mathrm{deg})$. The difference between the two distributions is statistically significant $\left(M_{1}-M_{2}=3.63 \mathrm{deg}, t=227, \mathrm{df}=125\right.$, $\mathrm{p}<.001)$.

\section{Statistical test of change in pointer settings}

Only runs with switching were used in this test. The comparison was between pointer settings in Part 1 and pointer settings in Part 3. Combining data for three Ss, there were 36 pointer settings in each group. The mean pointer setting in Part 1 was $13.83 \mathrm{deg}$ $(\mathrm{SD}=1.58 \mathrm{deg})$. The mean pointer setting in Part 3 was $13.45 \mathrm{deg}$ $(\mathrm{SD}=1.58 \mathrm{deg})$. The difference is not statistically significant $\left(M_{1}-M_{2}=0.38\right.$ deg, $t=1.12, d f=35,0.2<p<0.5$.

Comparison between pointer settings on peripheral targets at $5 \mathrm{deg}$ and $10 \mathrm{deg}$

Only runs with switching were used for this test. The comparison was between pointer settings in Part 1 and pointer settings in Part 4. Combining data for three Ss gave 36 pointer settings in each group. The mean pointer setting in Part 1 was $13.64 \mathrm{deg}(\mathrm{SD}=1.44 \mathrm{deg})$. The mean pointer setting in Part 4 was $9.37 \mathrm{deg}(\mathrm{SD}=1.14 \mathrm{deg})$. The difference is statistically significant $\left(M_{1}-M_{2}=4.27 \mathrm{deg}, \mathrm{t}=36, \mathrm{df}=35, \mathrm{p}<.001\right)$.

\section{Summary of results}

The parametric adjustment, which caused a change of $3.63 \mathrm{deg}$ in the magnitude of the initial saccade, had no effect on S's localization of the peripheral target. Comparison of pointer settings on a target at $10 \mathrm{deg}$ with pointer settings on a target at $5 \mathrm{deg}$ showed that, if the parametric adjustment had had a large effect on localization of the peripheral target, pointer settings would have revealed the effect.

Reports of subjective experience support the finding that visual localization is not affected by this type of parametric adjustment. In a series of exploratory experiments over a period of about one year, with extensive interrogation of naive $S$ s and discussion among practiced $\mathrm{Ss}$, we have been unable to obtain any reliable indication of even a momentary shift in localization of the peripheral target during parametric adjustment of saccadic eye movements.

\section{DISCUSSION}

Eye movements and judgments of visual extent

These results indicate that eye movements contribute nothing to perceived visual directions or to visual metric judgments, except insofar as they enable the observer to achieve clear (foveal) vision for all portions of the visual display.

Yarbus (1967) has reported experiments in which $S$ was required to make metric comparisons among portions of a complex figure when the figure was stablized on S's retina. Yarbus found that S's judgments were slow and inaccurate under these 
conditions, and concluded that: "Macromovements of the eye (large saccades) are necessary usually for the solution of certain problems (visual assessment of proportion and length, comparison of areas and angles, etc.)." The present results indicate that gross eye movements are necessary in these situations not because the movements themselves contribute any metric information, but because the human visual system is so constructed that it can best attack the problem by rapidly shifting fixation from one part of the test figure to another. With only a few seconds of multiple-fixation scanning, man's highly mobile eye can provide an array of visual information which is the functional equivalent of what would be seen by an eye with, say, 20 or 30 foveae.

There is also another factor which may have been operative in the Yarbus experiments: $\mathrm{S}$ may have found it disconcerting and confusing when his fixation reflex, elicited by the implied instruction to examine various portions of the test figure, failed to result in a change in the point of fixation. If this factor was an important contributor to the performance decrement, then it may be that Ss would improve with practice in making metric judgments on a stabilized retinal image.

\section{Eye movements, local signs, and visual localization}

In the theory of local signs as formulated by Lotze in 1852 , it was the eye movement and not the perceived visual direction that was tied to retinal locus. To quote Boring: "Lotze appealed to the innate mechanism by which we tend to fixate what comes into attention. The fixation of an object that lies in the periphery of the visual field requires a movement of the eyes and, moreover, a different movement for every point of the periphery. Experientially these movements ... furnish the visual local signs."

The present results show that, on the contrary, what is stable is the relation between retinal locus and perceived visual direction, while the magnitude of the eye movement is labile and in some sense "acquired by experience."

What then determines the magnitude and direction of a large saccade? First of all, the decision as to where to move the eyes may be determined by a verbal instruction, by an intent to search the visual field, by a quick motion perceived in the extreme periphery of the field of view-or as in the present experiment, by a definite and visible peripheral target. It seems likely that the neurological basis of control is different for each of these contingencies; and the list is not intended to be exhaustive. In the present experiment, then, we have investigated only one subspecies of macrosaccades.

A second class of factors includes the more peripheral determinants which are probably compensated by parametric feedback: fatigue of the extraocular muscles, metabolic state of the organism, starting position of the eye movement, success or failure of previous efforts to carry out a similar change of fixation, etc. The advantage of parametric feedback as a control principle is that the system does not need to monitor any of these variables: it need see only the momentary state of parametric adjustment. (The corresponding disadvantage is that an error must be made before a correction can be initiated.) Our results suggest that the human oculomotor system relies heavily on parametric feedback in control of large saccades, for it is capable of initiating a $50 \%$ parametric adjustment in only a few seconds.

The fact that a parametric adjustment of saccadic eye movements does not affect visual localization should not be taken as an indication that the link between a retinal locus and its associated visual direction is rigid and innate. Binocular unification of disparate images is one situation in which that link is modified in the interests of veridical perception; another is the perceptual adaptation to aniseikonia, in which retinal images differing in size are somehow combined to form a single percept. Still another example is a perceptual distortion such as the Muller-Lyer figure, in which there is a measurable difference between the metric of the retinal image and the corresponding percept. Nevertheless, the results of Harris (1965), Rock (1966) and others indicate that visual perceptions are stable and rigid in comparison with the proprioceptive and kinesthetic perceptual systems; and the present results confirm and extend that finding by showing that the oculomotor control system also gives way when placed in conflict with a visual percept.

\section{REFERENCES}

BORING, E. G. A history of experimental psychology. New York-London: D. Appleton-Century Co., Inc., 1929.

COOPER, S., \& DANIEL, P. M. Muscle spindles in human extrinsic eye muscles. Brain, 1949, 72, 1-24.

DODGE, R., \& CLINE, T. S. The angle velocity of eye movements. Psychol. Rev., 1901, 8, 145-157.

FENDER, D. H., \& NYE, P. W. An investigation of the mechanisms of eye movement control. Kybernetik, 1961, 1, 81-88.

GRANIT, R. Receptors and sensory perception. New Haven: Yale Univ. Press, 1955, pp. $191 \mathrm{ff}$.

HARRIS, C. S. Perceptual adaptation to inverted, reversed, and displaced vision. Psychol Rev., 1965, 72, 419-444.

KRAMER, M. E. Clinical orthoptics. (2nd ed.) St. Louis: The C. V. Mosby Co., 1953, pp. $464 \mathrm{ff}$.

LOTZE, H. Medicinische psychologie oder physiologie der seele. Leipzig, 1852.

LUDVIGH, E. Control of ocular movements and visual interpretation of environment. A.M.A. Arch. Ophthal., 1952, 48, 442-448.

McLAUGHLIN, S. C. Parametric adjustment in saccadic eye movements. Percept. \& Psychophys, 1967, 2, 359-362.

McLAUGHLIN, S. C., \& WEBSTER, R. G. Changes in straight-ahead eye position during adaptation to wedge prisms. Percept. \& Psychophys., 1967, 2,3644 .

McLAUGHLIN, S. C., \& KELLY, M. H., Jr. Parametric feedback in pursuit movements of the eyes. Paper read before the Optical Society of America, Washington, D. C., 14 March, 1968.

ROCK, I. The nature of perceptual adaptation. New York: Basic Books, 1966.

TOROK, N., GUILLEMIN, V., BARNOTHY, M. M. Photoelectric nystagmography. Ann. Otol, and Laryngol, 1951, 60, 917-926.

YARBUS, A. L. Eye movements and vision. New York: Plenum Press, 1967, pp. $200 \mathrm{ff}$.

\section{NOTES}

1. A shortened version of this paper was presented to the Optical Society of America, 12 October, 1967, in Detroit. (J. Opt. Soc. Amer., 1967, 57, 1425A.)

2. This research was supported by research grants from the National Science Foundation, the U.S. Army Medical Research and Development Command, and the Public Health Service (National Institute of Neurological Diseases and Blindness).

3. Now with American Aviation Corp., Wilmington, Mass.

4. Now at the Eye Research Foundation, 8710 Old Georgetown Road, Bethesda, Maryland.

5. The use of these horizontal dark areas was suggested by Mr. Brian Madden, Research Assistant, Tufts University.

(Accepted for publication March 5, 1968.) 